\title{
Des outils chimiques pour la biologie
}

\section{Prix Nobel de chimie 1987 : Jean-Marie Lehn, Donald J. Cram, Charles J. Pedersen}

La notion de " complémentarité " en biologie s'étend à toutes les interactions intermoléculaires spécifiques : celle d'une protéine avec un motif d'ADN, d'un ligand avec son transporteur ou son récepteur, d'un substrat avec une enzyme, d'un antigène avec un anticorps, etc. Les deux molécules qui forment un complexe doivent être complémentaires l'une de l'autre en terme de forme et de taille (comme une clé et sa serrure) et de sites de liaison. Sur cette base, il doit être possible, si on sait maîtriser la synthèse de macromolécules de formes définies, de créer des récepteurs " complémentaires " d'une grande diversité de ligands. Si ces "récepteurs" sont dotés de propriétés lipophiliques, ils pourront jouer le rôle de transporteurs transmembranaires et, s'ils contiennent un groupement actif, pourront catalyser, comme une enzyme, la modification du substrat lié. Les structures macropolycycliques sont particulièrement bien adaptées à la création de tels récepteurs synthétiques : elles peuvent en effet former des macromolécules comportant des cavités pouvant interagir, sur toute leur surface, avec des ligands. Le récepteur synthétique est appelé cryptand, formant un cryptate lorsqu'il est lié, au niveau de sa cavité centrale (la crypte) avec le substrat. Les applications possibles de ces molécules-outils sont multiples en catalyse chimique aussi bien qu'en biologie. Leur affinité pour différents types d'ions ou de petites molécules en pourraient faire des " détoxifiants " puissants en cas de contamination par des isotopes radioactifs ou d'intoxications diverses. On peut envisager de créer de véritables enzymes artificielles d'intérêt industriel. La combinaison de membranes artificielles et de vésicules dans lesquelles on peut inclure des structures constituant d'intéressants modèles de canaux ioniques, des catalyseurs macromoléculaires et des transporteurs, permet d'ima- giner la constitution de microréacteurs... voire de cellules artificielles. C'est donc un domaine nouveau, extrêmement riche en perspectives fondamentales et en possibilités d'applications, que créent ces nouveaux outils qu'imaginent et que construisent les chimistes. Les trois scientifiques auxquels le prix Nobel a été attribué, deux Américains et un Français originaire de Strasbourg (Jean-Marie Lehn, 48 ans, professeur au Collège de France), ont, à des titres divers, été à l'origine de cette nouvelle chimie des cryptates. Pedersen et Cram ont, tout d'abord, imaginé et synthétisé les " molécules en couronne ", planes, pouvant lier un ligand en leur centre. Lehn a donné une troisième dimension à ces molécules, transformant les couronnes en " cages".

A.K.

1. Lehn JM. Supramolecular chemistry : receptors, catalysts and carriers. Science 1985 ; $227: 849-56$
L'ADN complémentaire du messager codant pour le récepteur des minéralocorticoïdes a été cloné par le laboratoire de R. Evans [1] et sa séquence a été déterminée, permettant de déduire la structure protéique du récepteur. La concentration tissulaire de ce récepteur est très faible, si bien qu'il n'a jamais pu être totalement purifié. Des expériences déjà anciennes avaient néanmoins indiqué que le récepteur des minéralocorticoïdes (RM) pouvait fixer les glucocorticoïdes, suggérant que les récepteurs pour ces deux types d'hormone sont des protéines voisines. C'est donc par hybridation croisée avec une teur des glucocorticoïdes (RG) qu'a pu être isolé le clone d'ADNc du RM. Il code pour une protéine de 984 acides aminés (soit $107 \mathrm{kDa}$ ) dont la région de liaison à l'ADN a $94 \%$ d'homologie avec celle du RG, ce pourcentage étant de $57 \%$ pour la région de liaison de l'hormone. Le récepteur lie l'aldostérone et divers glucocorticoïdes avec la même affinité $\left(K_{D}=1,3 \mathrm{nM}\right)$. Le RM active la transcription contrôlée par le LTR (long terminal repeat) du virus de la tumeur mammaire de souris MMTV, en présence d'aldostérone aussi bien que de dexaméthasone (agoniste des glucocorticoïdes). Le RG, en revanche, n'active la transcription à partir de ce même promoteur qu'en présence de dexaméthasone. Le RM semble donc être aussi un récepteur à haute affinité des glucocorticoïdes (et, à plus faible affinité, de la progestérone), ce qui pourrait représenter en fait son rôle principal dans des tissus non rénaux insensibles à l'aldostérone et exprimant cependant le messager du RM, tels l'hippocampe. Il faut d'ailleurs noter que l'affinité pour les glucocorticoïdes du RM est bien plus grande $\left(K_{D}=\right.$ $1,3 \mathrm{nM})$ que celle du $\mathrm{RG}$ déjà caractérisé $\left(K_{D}=20-40 \mathrm{nM}\right)$.

[1. Azziza JL, et al. Science 1987 ; 237: 268-75] 